\title{
Non-existence of antipodal cages of even girth
}

\author{
Slobodan Filipovski* \\ University of Primorska \\ Koper, Slovenia \\ slobodan.filipovski@famnit.upr.si
}

\begin{abstract}
The Moore bound $M(k, g)$ is a lower bound on the order of $k$-regular graphs of girth $g$ (denoted $(k, g)$-graphs). The excess $e$ of a $(k, g)$-graph of order $n$ is the difference $n-M(k, g)$. A $(k, g)$-cage is a $(k, g)$-graph with the fewest possible number of vertices, among all $(k, g)$-graphs. A graph of diameter $d$ is said to be antipodal if, for any vertices $u, v, w$ such that $d(u, v)=d$ and $d(u, w)=d$, it follows that $d(v, w)=d$ or $v=w$. In [4] Biggs and Ito proved that any $(k, g)$-cage of even girth $g=2 d \geq 6$ and excess $e \leq k-2$ is a bipartite graph of diameter $d+1$. In this paper we treat $(k, g)$-cages of even girth and excess $e \leq k-2$. Based on a spectral analysis we give a relation between the eigenvalues of the adjacency matrix $A$ and the distance matrix $A_{d+1}$ of such cages. Moreover, following the methodology used in [4] and [13], we prove the non-existence of the antipodal $(k, g)$-cages of excess $e$, where $k \geq e+2 \geq 4$ and $g=2 d \geq 14$.
\end{abstract}

Keywords: $k$-regular graphs, antipodal cages, excess, multiplicities

\section{Introduction}

A $(k, g)$-graph is a $k$-regular graph having girth $g . \mathrm{A}(k, g)$-cage is a $(k, g)$-graph of smallest order. The Cage Problem or Degree/Girth Problem calls for finding cages; Tutte was the first to study $(k, g)$-cages, [16]. A $(k, g)$-graph exists for any pair $(k, g)$, where $k \geq 2$ and $g \geq 3$, see [8] and [14]. It is well known that the $(k, g)$-graphs have at least $M(k, g)$ vertices, where

$$
M(k, g)=\left\{\begin{array}{lr}
1+k+k(k-1)+\cdots+k(k-1)^{(g-3) / 2}, & g \text { odd } \\
2\left(1+(k-1)+\cdots+(k-1)^{(g-2) / 2}\right), & g \text { even }
\end{array}\right.
$$

If $G$ is a $(k, g)$-graph of order $n$, then we define the excess $e$ of $G$ to be $n-M(k, g)$. The graphs whose orders are equal to $M(k, g)$ (excess 0$)$ are called Moore graphs. Their

*Supported in part by the Slovenian Research Agency (research program P1-0285 and Young Researchers Grant). 
classification has been completed except for the case $k=57$ and $g=5$. The Moore graphs exist if $k=2$ and $g \geq 3, g=3$ and $k \geq 2, g=4$ and $k \geq 2, g=5$ and $k=2,3,7$, or $g=6,8,12$ and a generalized $n$-gon of order $k-1$ exists, see [1], [7] and [9].

The following three results concern the graphs of even girth.

Theorem 1.1 (Biggs and Ito [4]) Let $G$ be $a(k, g)$-cage of girth $g=2 d \geq 6$ and excess $e$. If $e \leq k-2$, then $e$ is even and $G$ is bipartite of diameter $d+1$.

It is known that these graphs are partially distance-regular. More about almost-distanceregular graphs, see [5]. For the next theorem, let $D(k, 2)$ denote the incidence graph of a symmetric $(v, k, 2)$-design.

Theorem 1.2 (Biggs and Ito [4]) Let $G$ be $a(k, g)$-cage of girth $g=2 d \geq 6$ and excess 2. Then $g=6, G$ is a double-cover of $D(k, 2)$, and $k \not \equiv 5,7(\bmod 8)$.

Theorem 1.3 (Jajcayová, Filipovski and Jajcay [12]) Let $k \geq 6$ and $g=2 d>6$. No $(k, g)$-graphs of excess 4 exist for parameters $k, g$ satisfying at least one of the following conditions:

1) $g=2 p$, with $p \geq 5$ a prime number, and $k \not \equiv 0,1,2(\bmod p)$;

2) $g=4 \cdot 3^{s}$ such that $s \geq 4$, and $k$ is divisible by 9 but not by $3^{s-1}$;

3) $g=2 p^{2}$, with $p \geq 5$ a prime number, and $k \not \equiv 0,1,2(\bmod p)$ and $k$ even;

4) $g=4 p$, with $p \geq 5$ a prime number, and $k \not \equiv 0,1,2,3, p-2(\bmod p)$;

5) $g \equiv 0(\bmod 16)$, and $k \equiv 3(\bmod g)$.

Let $k \geq 4, g=2 d \geq 6$ and let $G$ be a $(k, g)$-cage of excess $e \leq k-2$ and order $n$. Due to Theorem 1.1, we conclude that $G$ is a bipartite graph of diameter $d+1$. Let $A$ be its adjacency matrix. For the integers $i$ with $0 \leq i \leq d+1$, the $i$-distance matrix $A_{i}$ of $G$ is an $n \times n$ matrix such that the entry in position $(u, v)$ is 1 if the distance between the vertices $u$ and $v$ is $i$, and zero otherwise. Using the spectral considerations as in [10], in Section 2 we prove that the eigenvalues of $A\left(A_{1}\right)$, other than $\pm k$, are the roots of the polynomial $H_{d-1}(x)+\lambda$; Theorem 2.3. Here, $H_{d-1}$ is the Dickson polynomial of the second kind with parameter $k-1$ and degree $d-1$, and $\lambda$ is an eigenvalue of the distance matrix $A_{d+1}$.

A graph of diameter $d$ is said to be antipodal if, for any vertices $u, v, w$ such that $d(u, v)=d$ and $d(u, w)=d$, it follows that $d(v, w)=d$ or $v=w$, (see [3]).

Among the trivially antipodal graphs let us mention the $n$-dimensional cubes $Q_{n}$. These graphs are bipartite and have the antipodal property, since every vertex of $Q_{n}$ has a unique vertex at maximum distance from it. Also, for $n \geq 2$, the complete bipartite graph $K_{n, n}$ is antipodal. Here the antipodal partition is the same as the bipartition. The dodecahedron is an example of trivially antipodal, but not bipartite graph. Examples of graphs which are non-trivially antipodal and not bipartite are the complete tripartite graphs $K_{n, n, n}$, which have diameter 2, and the line graph of Petersen's graph, which has diameter 3. Motivated by Theorem 1.4, in this paper we address the question of the existence of the antipodal $(k, g)$-cages of even girth and excess $e \leq k-2$. Employing the methodology used in [2], 4] and [13], we prove the non-existence of the antipodal $(k, g)$-cages of excess $e$, with $k \geq e+2 \geq 4$ and $g=2 d \geq 14$; Theorem 4.2 . 
Theorem 1.4 (Bannai and Ito [2]) For $d \geq 3$, there exist no antipodal regular graphs with diameter $d+1$ and girth $2 d+1$.

\section{On $(k, g)$-cages of even girth and excess $e \leq k-2$}

Let $k, g, d$ and $e$ be positive integers such that $k \geq e+2$ and $g=2 d \geq 6$. Let $G$ be a $(k, g)$-cage of excess $e$; Theorem 1.1 asserts that $e$ is even and $G$ is a bipartite graph of diameter $d+1$. Let $f=\{u, v\}$ be an arbitrary edge of $G$. Let $\mathcal{T}_{u}$ be the subgraph of $G$ induced by the set of vertices $x \in V(G)$ such that $d(u, x) \leq \frac{g-2}{2}$ and $d(v, x)=d(u, x)+1$. It is easy to see that $\mathcal{T}_{u}$ is a tree of depth $\frac{g-2}{2}$ rooted at $u$. In the same way we can define the tree $\mathcal{T}_{v}$ to be the subgraph of $G$ induced by the set of vertices $x \in V(G)$ such that $d(v, x) \leq \frac{g-2}{2}$ and $d(u, x)=d(v, x)+1$. Since $G$ is of girth $g=2 d$, the trees $\mathcal{T}_{u}$ and $\mathcal{T}_{v}$ are disjoint. Let $\mathcal{T}_{u v}$ be the union of the trees $\mathcal{T}_{u}$ and $\mathcal{T}_{v}$ and the edge $f$. We call the graph $\mathcal{T}_{\text {uv }}$ a Moore tree of $G$ rooted at $f$. The graph $G$ must contain $e$ additional vertices $w_{1}, w_{2}, \ldots, w_{e-1}, w_{e}$, which do not belong to $\mathcal{T}_{u v}$, that is, $d\left(w_{i}, u\right)>\frac{g-2}{2}$ and $d\left(w_{i}, v\right)>\frac{g-2}{2}$, for each $1 \leq i \leq e$. We call these vertices the excess vertices with respect to $f$ and denote this set $X_{f}=\left\{w_{1}, w_{2}, \ldots, w_{e-1}, w_{e}\right\}$; we call the edges not contained in the Moore tree of $G$ horizontal edges. Since $G$ is a bipartite graph, it contains no odd cycle; consequently there exists no edge between the excess vertices of the same partite set. Moreover, in order to balance the Moore tree $\mathcal{T}_{u v}$ and paring out the horizontal edges of $G$, we easily observe that half $\left(\frac{e}{2}\right)$ of the excess vertices belong to the first, and the other half to the second partite set of $G$. It implies that for each vertex of $V(G)$ there exist exactly $\frac{e}{2}$ vertices at distance $d+1$ from it.

In order to study the spectral properties of $G$, we define the following polynomials:

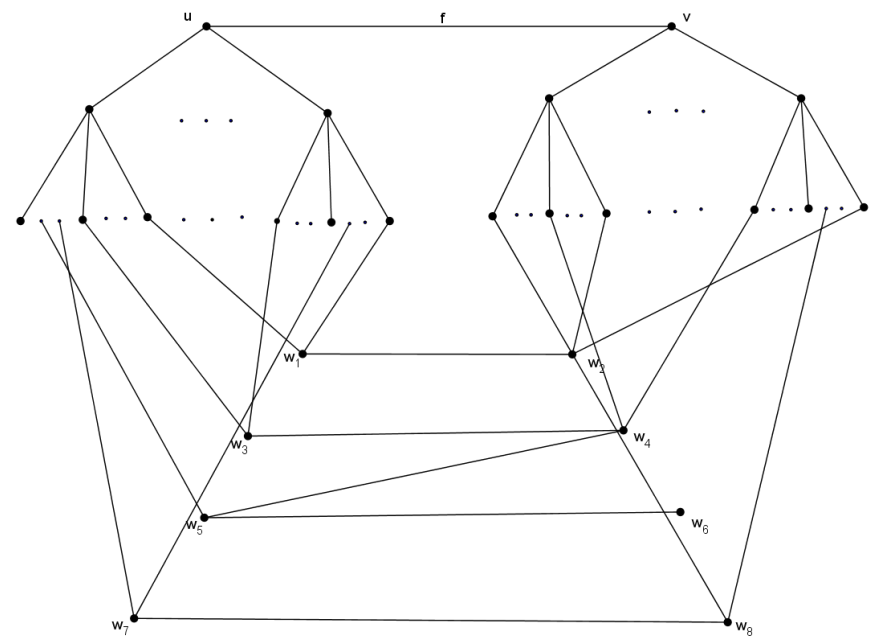

Figure 1: The Moore tree and some of the horizontal edges in a potential $(k, 6)$-graph of excess 8

$$
G_{0}(x)=1, G_{1}(x)=x+1 ;
$$




$$
\begin{gathered}
F_{0}(x)=1, F_{1}(x)=x, F_{2}(x)=x^{2}-k ; \\
H_{-2}(x)=-\frac{1}{k-1}, H_{-1}(x)=0, H_{0}(x)=1, H_{1}(x)=x ; \\
P_{i+1}(x)=x P_{i}(x)-(k-1) P_{i-1}(x) \text { for } \begin{cases}i \geq 1, & \text { if } P_{i}=G_{i}, \\
i \geq 2, & \text { if } P_{i}=F_{i}, \\
i \geq 1, & \text { if } P_{i}=H_{i} .\end{cases}
\end{gathered}
$$

The above defined polynomials have a close connection to the properties of a graph $G$. Namely, for $l<g$, the element $\left(F_{l}(A)\right)_{x, y}$ counts the number of paths of length $l$ joining vertices $x$ and $y$ of $G$. Moreover, $G_{l}(A)$ counts the number of paths of length at most $l$ joining pairs of vertices in $G$. For more information about these polynomials see [15].

The next lemma is a generalization on Lemma 5 from [10], where it is considered cages of even girth and excess 4 .

Lemma 2.1 Let $k \geq e+2$ and $g=2 d \geq 6$, and let $G$ be $a(k, g)$-cage of excess e. If $A$ is the adjacency matrix of $G$, then

$$
F_{d}(A)=k A_{d}-A A_{d+1}
$$

Proof. Let $f=\{u, v\}$ be a base edge of the Moore tree and let $f_{1}=\left\{w_{1}, w_{2}\right\}, f_{2}=$ $\left\{w_{3}, w_{4}\right\}, \ldots, f_{\frac{e}{2}}=\left\{w_{e-1}, w_{e}\right\}$ be the edges of the subgraph induced by $X_{f}$. Also, let us assume that $d\left(u, w_{1}\right)=d\left(u, w_{3}\right)=\ldots=d\left(u, w_{e-1}\right)=d$ and $d\left(u, w_{2}\right)=d\left(u, w_{4}\right)=\ldots=$ $d\left(u, w_{e}\right)=d+1$. Let $l_{i}$ be the number of edges between $w_{i}$ and the leaves of $\mathcal{T}_{u}$ and $\mathcal{T}_{v}$, where $1 \leq i \leq e$. We consider the case when the excess vertices do not share common neighbour among the leaves of $\mathcal{T}_{u}$ and $\mathcal{T}_{v}$. The opposite case one can prove in a similar way. By the definition of $F_{i}(x)$, we have $\left(F_{d}(A)\right)_{u, w_{i}}=l_{i}$, for each odd $i, 1 \leq i \leq e-1$. Considering the vertices at distance $d$ from $u$, there are also the $(k-1)^{d-1}$ leaves of $\mathcal{T}_{v}$. For $l_{2}+l_{4}+\ldots+l_{e}$ of these vertices, there exist $k-1$ paths of length $d$ from $u$ to them. Namely, they are the vertices adjacent to $w_{2}, w_{4}, \ldots, w_{e-2}$ or $w_{e}$. For all the other leaves, there are $k$ paths between them and $u$. Thus, $\left(F_{d}(A)\right)_{u, s}=0$ if $d(u, s) \neq d,\left(F_{d}(A)\right)_{u, s}=k$ if $s$ is a leaf of $\mathcal{T}_{v}$ and not adjacent to $w_{2}, w_{4}, \ldots, w_{e},\left(F_{d}(A)\right)_{u, s}=k-1$ if $s$ is a leaf of $\mathcal{T}_{v}$ and adjacent to one of $w_{2}, w_{4}, \ldots, w_{e}$, and $\left(F_{d}(A)\right)_{u, w_{i}}=l_{i}$, for each odd $i ; 1 \leq i \leq e-1$. For the matrix $k A_{d}$ we have $\left(k A_{d}\right)_{u, s}=k$ if $d(u, s)=d$ and $\left(k A_{d}\right)_{u, s}=0$ if $d(u, s) \neq d$. Now, let $s$ be a vertex of $G$ such that $d(u, s)=d$ and $s$ is adjacent to one of $w_{2}, w_{4}, \ldots, w_{e}$. If $s$ is a vertex among the vertices $w_{1}, w_{3}, \ldots, w_{e-1}$, then it is easy to see that $\left(A A_{d+1}\right)_{u, s}=k-l_{i}$. On the other hand, since $s$ is adjacent to $\mathcal{T}_{u}$ through $k-2$ different horizontal edges, it follows that, between the $k-1$ branches of $\mathcal{T}_{u}$, there exists one sub-branch that is not adjacent to $s$ through a horizontal edge. Let $s_{1}$ be the root of that sub-branch. Then, $d\left(s, s_{1}\right)=d+1$ and $d\left(u, s_{1}\right)=1$, which implies $(A)_{u, s_{1}}=1$ and $\left(A_{d+1}\right)_{s_{1}, s}=1$. Let $s_{i}, 2 \leq i \leq \frac{e}{2}$ be the remaining vertices at distance $d+1$ from $s$. Because all neighbours of $u$, except $s_{1}$, are at distance smaller than $d+1$ from $s$, we have $(A)_{u, s_{i}}=0$ and $\left(A_{d+1}\right)_{s_{i}, s}=1$, for $2 \leq i \leq \frac{e}{2}$. Thus $\left(A A_{d+1}\right)_{u, s}=1$. If $s$ is a vertex of $G$ such that $d(u, s)=d$ and $s$ is not adjacent to $w_{2}, w_{4}, \ldots, w_{e}$, then the distance between $s$ and the neighbours of $u$ is $d-1$. In this case, $\left(A A_{d+1}\right)_{u, s}=0$. If $d(u, s) \neq d$, then the distance between $s$ and the neighbours of $u$ is different from $d+1$, and therefore $\left(A A_{d+1}\right)_{u, s}=0$. The required identity follows from summing up the above conclusions. 
Based on the previous lemma and the properties of the polynomials $G_{i}, H_{i}$ and $F_{i}$, we obtain the next two results. Theorem 2.3 is the main result in this section; it gives a relationship between the eigenvalues of the matrices $A$ and $A_{d+1}$. We omitted their proofs because they follow analoglously like in Lemma 6 and Theorem 7 from [10].

Lemma 2.2 Let $k \geq e+2 \geq 4$ and $g=2 d \geq 6$, and let $G$ be a $(k, g)$-cage of excess $e$. If $A$ is the adjacency matrix of $G$ and $J$ is the all-ones matrix, then

$$
k J=(A+k I)\left(H_{d-1}(A)+A_{d+1}\right) .
$$

Theorem 2.3 If $\theta(\neq \pm k)$ is an eigenvalue of $A$, then

$$
H_{d-1}(\theta)=-\lambda,
$$

where $\lambda$ is an eigenvalue of $A_{d+1}$.

\section{Spectral analysis of the antipodal cages of even girth and small excess}

In this section we study the spectral properties of the antipodal $(k, g)$-cages of even girth $g=2 d \geq 6$ and excess at most $k-2$. Let $G$ be such graph, $A$ be its adjacency matrix and let $n$ be the order of $G$. Recall, $G$ is a bipartite graph of diameter $d+1$. Let $V_{1}$ and $V_{2}$ be the partitions of $G$. If $d$ is an even number, then any two vertices of $V(G)$ at distance $d+1$ belong to a different partite set. Clearly, this case is not possible considering antipodal bipartite graphs. Therefore, for the rest of the paper we assume $d$ odd. Since for each vertex $u \in V(G)$ there exist exactly $\frac{e}{2}$ vertices at diameter distance $d+1$, (they are the excess vertices of the same partite set), we observe that the antipodal

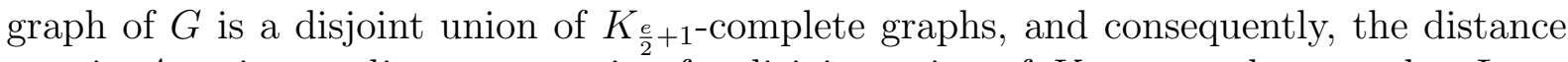
matrix $A_{d+1}$ is an adjacency matrix of a disjoint union of $K_{\frac{e}{2}+1}$-complete graphs. Let $c$ be the number of such complete graphs. Obviously $c=\frac{2 n}{e+2}$. The spectrum of the disjoint union of $c$ complete graphs of order $\frac{e}{2}+1$ is known and determined by $\left\{\left(\frac{e}{2}\right)^{c},(-1)^{n-c}\right\}$ (see Propos. 6 in [6]). Applying this result in Theorem 2.3, we are in a position to determine the spectrum of $A$.

Theorem 3.1 If $\theta(\neq \pm k)$ is an eigenvalue of $A$, then

$$
H_{d-1}(\theta)-\epsilon=0,
$$

where $\epsilon=-\frac{e}{2}, 1$.

The roots of $H_{d-1}(x)$ are equal to $2 \sqrt{k-1} \cos \frac{i \pi}{d}$ for $i=1, \ldots, d-1$, (see [15]). Therefore we assume $x=-2 \sqrt{k-1} \cos \phi, 0<\phi<\pi$. Let $s=\sqrt{k-1}$. Then we have

$$
H_{d-1}(x)=(-s)^{d-1} \frac{\sin d \phi}{\sin \phi}
$$


Putting $\phi=\frac{i \pi-\alpha}{d}$, as suggested in [2] and [4], we transform the equation (3) as follows

$$
\sin \alpha-\eta_{i} s^{-d+1} \sin \left(\frac{i \pi-\alpha}{d}\right)=0
$$

where $\eta_{i}=\epsilon(-1)^{d+i}$. The following result follows similarly as Lemma 3.3 from [4] and Lemma 2.2 from [13].

Lemma 3.2 The equation (3) has $d-1$ distinct roots $\theta_{1}<\theta_{2}<\ldots<\theta_{d-1}$, with $\theta_{i}=$ $-2 s \cos \phi_{i},\left(0<\phi_{i}<\pi\right)$. If we set $\phi_{i}=\frac{i \pi-\alpha_{i}}{d}$ then

$$
\begin{gathered}
0<\alpha_{i}<\min \left\{s^{-d+1} \phi_{i}, s^{-d+1}\left(\pi-\phi_{i}\right)\right\} \text { if } \eta_{i}=1 \\
\max \left\{-s^{-d+1} \phi_{i},-s^{-d+1}\left(\pi-\phi_{i}\right)\right\}<\alpha_{i}<0 \text { if } \eta_{i}=-1 \\
0<\alpha_{i}<\min \left\{\frac{e}{2} s^{-d+1} \phi_{i}, \frac{e}{2} s^{-d+1}\left(\pi-\phi_{i}\right)\right\} \text { if } \eta_{i}=\frac{e}{2} \\
\max \left\{-\frac{e}{2} s^{-d+1} \phi_{i},-\frac{e}{2} s^{-d+1}\left(\pi-\phi_{i}\right)\right\}<\alpha_{i}<0, \text { if } \eta_{i}=-\frac{e}{2} .
\end{gathered}
$$

From the bounds of $\alpha_{i}$ we derive the bounds of $\phi_{i}$ as follows.

$$
\begin{gathered}
\frac{i \pi}{d+s^{-d+1}}<\phi_{i}<\frac{i \pi}{d} \text { if } \eta_{i}=1 ; \\
\frac{i \pi}{d}<\phi_{i}<\frac{i \pi}{d-s^{-d+1}} \text { if } \eta_{i}=-1 ; \\
\frac{i \pi}{d+\frac{e}{2} s^{-d+1}}<\phi_{i}<\frac{i \pi}{d} \text { if } \eta_{i}=\frac{e}{2} \\
\frac{i \pi}{d}<\phi_{i}<\frac{i \pi}{d-\frac{e}{2} s^{-d+1}} \text { if } \eta_{i}=-\frac{e}{2} .
\end{gathered}
$$

We claim that $\operatorname{tr}\left(A^{q}\right)=n\left(B_{d}^{q}\right)_{0,0}$ for $q=0,1, \ldots, 2 d-1$, where

$$
B_{D}=\left(\begin{array}{cccccccc}
0 & 1 & & & & & & \\
k & 0 & 1 & & & & 0 & \\
& k-1 & 0 & 1 & & & & \\
& & k-1 & 0 & 1 & & & \\
& & & & \ddots & \ddots & & \\
& & & & \ddots & & & \\
& 0 & & & & k-1 & 0 & k \\
& & & & & & k-1 & 0
\end{array}\right)
$$

is the $(D+1) \times(D+1)$ intersection matrix of a Moore bipartite graph of degree $k$, diameter $D$ and of girth $2 D$, (see [13]). If $q<g(G)$, the number of closed walks of length $q$ that start from a fixed vertex $u$ is independent of the vertex $u$ and the excess. Furthermore, the entry $\left(B_{\left\lceil\frac{g(G)}{2}\right\rceil}^{q}\right)_{0,0}$ gives this number, where $\left(B_{i}^{q}\right)_{0,0}$ is the $(0,0)$-entry of $B_{i}^{q}$, (see [11]). The number of closed walks of length $q$ in $G$ is given by $\operatorname{tr}\left(A^{q}\right)$. Since $G$ is a bipartite graph, it follows that $G$ contains no closed walk of odd length. Thus, $\operatorname{tr}\left(A^{q}\right)=n\left(B_{d}^{q}\right)_{(0,0)}$ for $q=1,3, \ldots, 2 d-3,2 d-1$. Moreover, since the girth of $G$ is $2 d$ we obtain $\operatorname{tr}\left(A^{q}\right)=n\left(B_{d}^{q}\right)_{(0,0)}$ for $q=0,1, \ldots, 2 d-1$. 
Theorem 3.3 Let $\theta$ be a root of $H_{d-1}(x)-\epsilon$. The multiplicity $m(\theta)$ of $\theta$ in $G, \theta \neq \pm k$, is given by

$$
m(\theta)=\frac{n e k(k-1) H_{d-2}(\theta)}{2 \epsilon\left(2 \epsilon+\frac{e}{2}-1\right) H_{d-1}^{\prime}(\theta)\left(k^{2}-\theta^{2}\right)} .
$$

Proof. In order to compute the multiplicity of an eigenvalue $\theta$ of $G$, we employ the same approach from [2], 44] and [13]. Let $\xi(x)=\left(x^{2}-k^{2}\right)\left(H_{d-1}(x)+\frac{e}{2}\right)\left(H_{d-1}(x)-1\right)$ and $\xi_{\theta}(x)=\frac{\xi(x)}{x-\theta}$. Since $\xi(A)=0$, it follows $m(\theta)=\frac{\operatorname{tr}\left(\xi_{\theta}(A)\right)}{\xi_{\theta}(\theta)}$.

As $\operatorname{deg}\left(H_{d-1}(x)\right)=d-1$ we obtain that $\operatorname{deg}\left(\xi_{\theta}(x)\right)=2 d-1$. Therefore, let us assume $\xi_{\theta}(x)=x^{2 d-1}+a_{2 d-2} x^{2 d-2}+\ldots+a_{1} x+a_{0}$. Hence,

$$
\operatorname{tr}\left(\xi_{\theta}(A)\right)=\operatorname{tr}\left(A^{2 d-1}\right)+a_{2 d-2} \operatorname{tr}\left(A^{2 d-2}\right)+\ldots+a_{1} \operatorname{tr}(A)+a_{0} \operatorname{tr}\left(I_{n}\right) .
$$

Since $\operatorname{tr}\left(A^{q}\right)=n\left(B_{d}^{q}\right)_{0,0}$ for $0 \leq q \leq 2 d-1$, we have

$$
\operatorname{tr}\left(\xi_{\theta}(A)\right)=n\left(\xi_{\theta}\left(B_{d}\right)\right)_{0,0} .
$$

The polynomial $\left(x^{2}-k^{2}\right) H_{d-1}(x)$ is a minimal polynomial of $B_{d}$, (see [15]). It implies

$$
\xi_{\theta}\left(B_{d}\right)=-\frac{e}{2} \frac{B_{d}^{2}-k^{2} I_{n}}{B_{d}-\theta I_{n}}
$$

Setting $L_{i+1}(x)=\frac{x^{2}-k^{2}}{x-\theta}\left(H_{i}(x)-H_{i}(\theta)\right)$ for $i=0, \ldots, d-1$, we get

$$
L_{d}\left(B_{d}\right)=-H_{d-1}(\theta) \frac{B_{d}^{2}-k^{2} I_{n}}{B_{d}-\theta I_{n}}=-\epsilon \frac{B_{d}^{2}-k^{2} I_{n}}{B_{d}-\theta I_{n}} .
$$

Therefore, $\xi_{\theta}\left(B_{d}\right)=\frac{e}{2 \epsilon} L_{d}\left(B_{d}\right)$.

Calculating the derivative of $(x-\theta) \xi_{\theta}(x)$, that is, $\left((x-\theta) \xi_{\theta}(x)\right)^{\prime}=\left(\left(x^{2}-k^{2}\right)\left(H_{d-1}(x)+\right.\right.$ $\left.\left.\frac{e}{2}\right)\left(H_{d-1}(x)-1\right)\right)^{\prime}$, we have $\xi_{\theta}(\theta)=\left(2 \epsilon+\frac{e}{2}-1\right) H_{d-1}^{\prime}(\theta)\left(\theta^{2}-k^{2}\right)$. Thus

$$
m(\theta)=\frac{n e}{2 \epsilon\left(2 \epsilon+\frac{e}{2}-1\right)} \frac{\left(L_{d}\left(B_{d}\right)\right)_{0,0}}{H_{d-1}^{\prime}(\theta)\left(\theta^{2}-k^{2}\right)} .
$$

In [13] was proven that $\left(L_{d}\left(B_{d}\right)\right)_{0,0}=-k(k-1) H_{d-2}(\theta)$. Substituting it in the previous expression we obtain

$$
m(\theta)=\frac{n e k(k-1)}{2 \epsilon\left(2 \epsilon+\frac{e}{2}-1\right)} \frac{H_{d-2}(\theta)}{H_{d-1}^{\prime}(\theta)\left(k^{2}-\theta^{2}\right)} .
$$

\subsection{Multiplicities as function of $\cos \phi$}

Let $\theta$ be a root of $H_{d-1}(x)-\epsilon$ and let $\theta=-2 s \cos \phi, 0<\phi<\pi$. We express the multiplicity of $\theta, m(\theta)$, as a function of $\cos \phi$. For that purpose we define the following functions $f(z), g_{1}(z), g_{2}(z)$ and $g_{3}(z)$.

$$
f(z)=\frac{4 s^{2}\left(1-z^{2}\right)}{k^{2}-4 s^{2} z^{2}}
$$




$$
\begin{gathered}
g_{1}(z)=\frac{k(k-1)\left(\sqrt{1-s^{-2 d+2}\left(1-z^{2}\right)}+s^{-d+1} z\right)}{d \sqrt{1-s^{-2 d+2}\left(1-z^{2}\right)}+s^{-d+1} z} ; \\
g_{2}(z)=\frac{k(k-1)\left(\sqrt{1-\frac{e^{2}}{4} s^{-2 d+2}\left(1-z^{2}\right)}-\frac{e}{2} s^{-d+1} z\right)}{d \sqrt{1-\frac{e^{2}}{4} s^{-2 d+2}\left(1-z^{2}\right)}-\frac{e}{2} s^{-d+1} z} ; \\
g_{3}(z)=\frac{k(k-1)\left(\sqrt{1-\frac{e^{2}}{4} s^{-2 d+2}\left(1-z^{2}\right)}+\frac{e}{2} s^{-d+1} z\right)}{d \sqrt{1-\frac{e^{2}}{4} s^{-2 d+2}\left(1-z^{2}\right)}+\frac{e}{2} s^{-d+1} z} .
\end{gathered}
$$

Lemma 3.4 For either value of $\epsilon$, if we set $\theta_{i}=-2 s \cos \phi_{i}$ for $1 \leq i \leq d-1$, then

$$
\begin{gathered}
m\left(\theta_{i}\right)=\frac{n e}{4 s^{2}\left(\frac{e}{2}+1\right)} f\left(\cos \phi_{i}\right) g_{1}\left(\eta_{i} \cos \phi_{i}\right), \text { if } \epsilon=1 ; \\
m\left(\theta_{i}\right)=\frac{n}{2 s^{2}\left(\frac{e}{2}+1\right)} f\left(\cos \phi_{i}\right) g_{2}\left(\cos \phi_{i}\right), \text { if } \epsilon=-\frac{e}{2} \text { and } i \text { is odd; } \\
m\left(\theta_{i}\right)=\frac{n}{2 s^{2}\left(\frac{e}{2}+1\right)} f\left(\cos \phi_{i}\right) g_{3}\left(\cos \phi_{i}\right), \text { if } \epsilon=-\frac{e}{2} \text { and } i \text { is even. }
\end{gathered}
$$

Proof. The derivative of $H_{d-1}(x)$ is computed in [13]. We have

$$
H_{d-1}^{\prime}\left(\theta_{i}\right)=\frac{(-s)^{d-1}(-1)^{i}}{2 s \sin ^{2} \phi_{i}}\left(d \cos \alpha_{i}+\eta_{i} s^{-d+1} \cos \phi_{i}\right)
$$

Substituting $H_{d-2}\left(\theta_{i}\right)=(-s)^{d-2}(-1)^{i+1} \frac{\sin \left(\phi_{i}+\alpha_{i}\right)}{\sin \phi_{i}}$ and $H_{d-1}^{\prime}\left(\theta_{i}\right)$ in $(5)$, we obtain

$$
m\left(\theta_{i}\right)=\frac{n e k(k-1) \sin \phi_{i} \sin \left(\phi_{i}+\alpha_{i}\right)}{\epsilon\left(2 \epsilon+\frac{e}{2}-1\right)\left(k^{2}-\theta_{i}^{2}\right)\left(d \cos \alpha_{i}+\eta_{i} s^{-d+1} \cos \phi_{i}\right)} .
$$

The equation (4) yields $\sin \left(\phi_{i}+\alpha_{i}\right)=\sin \phi_{i}\left(\cos \alpha_{i}+\eta_{i} s^{-d+1} \cos \phi_{i}\right)$. Hence

$$
m\left(\theta_{i}\right)=\frac{n e \sin ^{2} \phi_{i}}{\epsilon\left(2 \epsilon+\frac{e}{2}-1\right)\left(k^{2}-\theta_{i}^{2}\right)} \frac{k(k-1)\left(\cos \alpha_{i}+\eta_{i} s^{-d+1} \cos \phi_{i}\right)}{\left(d \cos \alpha_{i}+\eta_{i} s^{-d+1} \cos \phi_{i}\right)} .
$$

By equation (4) and Lemma 3.2, as $k, d \geq 3$, it follows that if $\eta_{i}=1$ or $\eta_{i}=\frac{e}{2}$ then $0<\alpha_{i}<\frac{\pi}{2}$. Similarly, if $\eta_{i}=-1$ or $\eta_{i}=-\frac{e}{2}$, then $-\frac{\pi}{2}<\alpha_{i}<0$. Therefore $\cos \alpha_{i}>0$, and thus, $\cos \alpha_{i}=\sqrt{1-\eta_{i}^{2} s^{-2 d+2}\left(1-\cos ^{2} \phi_{i}\right)}$. It implies

$m\left(\theta_{i}\right)=\frac{n e}{4 s^{2} \epsilon\left(2 \epsilon+\frac{e}{2}-1\right)} \frac{4 s^{2}\left(1-\cos ^{2} \phi_{i}\right)}{k^{2}-4 s^{2} \cos ^{2} \phi_{i}} \frac{k(k-1)\left(\sqrt{1-\eta_{i}^{2} s^{-2 d+2}\left(1-\cos ^{2} \phi_{i}\right)}+\eta_{i} s^{-d+1} \cos \phi_{i}\right)}{\left(d \sqrt{1-\eta_{i}^{2} s^{-2 d+2}\left(1-\cos ^{2} \phi_{i}\right)}+\eta_{i} s^{-d+1} \cos \phi_{i}\right)}$.

Using the formulas for $f, g_{1}, g_{2}$ and $g_{3}$ we get the desired result.

The following two lemmas concern the monotonicity of $f, g_{1}, g_{2}$ and $g_{3}$. The first lemma is given in [4] and [13] (Lemma 3.5 and Lemma 4.1). 
Lemma 3.5 For $k \geq 3$ and $|z|<1$ the function $f(z)$ is even and concave down.

Lemma 3.6 For $k \geq 3, d \geq 3$ and $|z|<1$, the monotonicity of $g_{1}(z), g_{2}(z)$ and $g_{3}(z)$ behave as follows.

(1) $g_{1}(z)$ is monotonic increasing;

(2) $g_{2}(z)$ is monotonic decreasing;

(3) $g_{3}(z)$ is monotonic increasing;

Proof.

(1) It is suffice to prove that $g_{1}^{\prime}(z)$ is positive on the interval $(-1,1)$. We have

$$
g_{1}^{\prime}(z)=\frac{k(k-1)(d-1) s^{-d+1}\left(1-s^{-2 d+2}\right)}{\sqrt{1+s^{-2 d+2}\left(-1+z^{2}\right)}\left(d \sqrt{1+s^{-2 d+2}\left(-1+z^{2}\right)}+s^{-d+1} z\right)^{2}}>0 .
$$

(2) In this case we prove that $g_{2}^{\prime}(z)$ is negative on the interval $(-1,1)$.

$$
g_{2}^{\prime}(z)=\frac{-\frac{e}{2} s^{-d+1} k(k-1)(d-1)\left(1-\frac{e^{2}}{4} s^{-2 d+2}\right)}{\sqrt{1+\frac{e^{2}}{4} s^{-2 d+2}\left(-1+z^{2}\right)}\left(d \sqrt{1+\frac{e^{2}}{4} s^{-2 d+2}\left(-1+z^{2}\right)}-\frac{e}{2} s^{-d+1} z\right)^{2}} .
$$

Since $k, d \geq 3$ and $k \geq e+2$, we easily conclude that $\frac{e^{2}}{4} s^{-2 d+2}<1$ and $\mid \frac{e^{2}}{4} s^{-2 d+2}(-1+$ $\left.z^{2}\right) \mid<1$.

(3) It follows from the same reasoning as (2).

\section{Main result}

Let $\lambda_{1}<\lambda_{2}<\ldots<\lambda_{d-1}$ be the roots of $H_{d-1}(x)+\frac{e}{2}$, and let $\mu_{1}<\mu_{2}<\ldots<\mu_{d-1}$ be the roots of $H_{d-1}(x)-1$.

Lemma 4.1 Let $\lambda_{1}, \ldots, \lambda_{d-1}$ and $\mu_{1}, \ldots, \mu_{d-1}$ be defined as above. If $k \geq 3$ and $d \geq 3$ is an odd number, then

(1) $m\left(\lambda_{i}\right)=m\left(\lambda_{d-i}\right)$ and $m\left(\mu_{i}\right)=m\left(\mu_{d-i}\right)$, for $1 \leq i \leq d-1$;

(2) $m\left(\mu_{2}\right)<m\left(\mu_{i}\right)$ for $3 \leq i \leq d-3$, and $m\left(\lambda_{1}\right)<m\left(\lambda_{i}\right)$, for $2 \leq i \leq d-2$.

PROOF.

(1) If $d$ is odd $H_{d-1}(-x)=H_{d-1}(x)$. Therefore $\theta$ is a root of $H_{d-1}(x)-\epsilon$, if and only if, $-\theta$ is a root of $H_{d-1}(x)-\epsilon$, (see [1]). Then $\lambda_{i}+\lambda_{d-i}=\mu_{i}+\mu_{d-i}=0$. By checking (5) and using $H_{d-2}(-x)=-H_{d-2}(x)$, we obtain $m\left(\lambda_{i}\right)=m\left(\lambda_{d-i}\right)$ and $m\left(\mu_{i}\right)=m\left(\mu_{d-i}\right)$ for each $1 \leq i \leq d-1$. 
(2) Since $\mu_{i}$ is a root of $H_{d-1}(x)-1$, we have $\epsilon=1$. According to Lemma 3.2 let us set $\mu_{i}=-2 s \cos \phi_{i}$, for $1 \leq i \leq d-1$. In this case $\eta_{i}=\epsilon(-1)^{d+i}=(-1)^{i+1}$. Since $-\mu_{2}=\mu_{d-2}$ we obtain $-\cos \phi_{2}=\cos \phi_{d-2}$. Now, for $3 \leq i \leq d-3$, we have $-\cos \phi_{2}=\cos \phi_{d-2}<\cos \phi_{i}<\cos \phi_{2}$. Since $f$ is even and concave down function we have

$$
f\left(\cos \phi_{2}\right)<f\left(\cos \phi_{i}\right) \text { for } 3 \leq i \leq d-3 .
$$

The inequality $\cos \phi_{i}<\left|\cos \phi_{2}\right|$ and the fact that $g_{1}(z)$ is a monotonic increasing function yield $g_{1}\left(\eta_{2} \cos \phi_{2}\right)=g_{1}\left(-\cos \phi_{2}\right)<g_{1}\left( \pm \cos \phi_{i}\right)$.

Therefore, for $3 \leq i \leq d-3$, we conclude

$$
m\left(\mu_{2}\right)=\frac{n e}{4 s^{2}\left(\frac{e}{2}+1\right)} f\left(\cos \phi_{2}\right) g_{1}\left(\eta_{2} \cos \phi_{2}\right)<\frac{n e}{4 s^{2}\left(\frac{e}{2}+1\right)} f\left(\cos \phi_{i}\right) g_{1}\left( \pm \cos \phi_{i}\right)=m\left(\mu_{i}\right) .
$$

We proceed similarly when $\lambda_{i}$ is a root of $H_{d-1}(x)+\frac{e}{2}$. In this case $\epsilon=-\frac{e}{2}$ and $\eta_{i}=\frac{e}{2}(-1)^{i}$. Again let $\lambda_{i}=-2 s \cos \phi_{i}$, for $1 \leq i \leq d-1$. Following the same reasoning as above we have $f\left(\cos \phi_{1}\right)<f\left(\cos \phi_{i}\right)$ for $2 \leq i \leq d-2$.

Now, let $i$ be an odd number such that $3 \leq i \leq d-2$. For such $i$ we note $\eta_{i}=$ $-\frac{e}{2}<0$. Hence $g_{2}(z)$ is a monotonic decreasing, and therefore, $\cos \phi_{i}<\cos \phi_{1}$ yields $g_{2}\left(\cos \phi_{1}\right)<g_{2}\left(\cos \phi_{i}\right)$. Thus, for odd $i$ such that $3 \leq i \leq d-2$ we have

$$
m\left(\lambda_{1}\right)=\frac{n}{2 s^{2}\left(\frac{e}{2}+1\right)} f\left(\cos \phi_{1}\right) g_{2}\left(\cos \phi_{1}\right)<\frac{n}{2 s^{2}\left(\frac{e}{2}+1\right)} f\left(\cos \phi_{i}\right) g_{2}\left(\cos \phi_{i}\right)=m\left(\lambda_{i}\right) .
$$

Since $m\left(\lambda_{i}\right)=m\left(\lambda_{d-i}\right)$ occurs $m\left(\lambda_{1}\right)<m\left(\lambda_{i}\right)$ for each $2 \leq i \leq d-2$.

Based on Lemma 4.1, we are ready to give the main result in this paper.

Theorem 4.2 Let $k \geq e+2 \geq 4$ and $g=2 d \geq 14$ be fixed. There exists no antipodal $(k, g)$-cage of excess e.

Proof. Since $m\left(\mu_{2}\right)<m\left(\mu_{i}\right)$ for $3 \leq i \leq d-3$ and $\mu_{1}+\mu_{d-1}=0$, we obtain that $\mu_{2}$ and $\mu_{d-2}=-\mu_{2}$ are either conjugate quadratic irrationals or integers. Therefore, $\mu_{2}^{2}$ is an integer. Analogously, $\lambda_{2}^{2}$ is an integer. Hence $\lambda_{2}^{2}-\mu_{2}^{2}$ is an integer number. By Lemma 3.2 we have

$$
\begin{aligned}
& -2 s \cos \frac{2 \pi}{d}<\mu_{2}<-2 s \cos \frac{2 \pi}{d-s^{-d+1}} \\
& -2 s \cos \frac{2 \pi}{d+\frac{e}{2} s^{-d+1}}<\lambda_{2}<-2 s \cos \frac{2 \pi}{d} .
\end{aligned}
$$

Then, as $\lambda_{2}^{2}>4 s^{2} \cos ^{2} \frac{2 \pi}{d}$ and $\mu_{2}^{2}<4 s^{2} \cos ^{2} \frac{2 \pi}{d}$, we have that $\lambda_{2}^{2}-\mu_{2}^{2}>0$. Furthermore, as $\lambda_{2}^{2}<4 s^{2} \cos ^{2} \frac{2 \pi}{d+\frac{e}{2} s^{-d+1}}$ and $\mu_{2}^{2}>4 s^{2} \cos ^{2} \frac{2 \pi}{d-s^{-d+1}}$, we have that

$$
\begin{aligned}
\lambda_{2}^{2}-\mu_{2}^{2} & <4 s^{2}\left(\cos ^{2} \frac{2 \pi}{d+\frac{e}{2} s^{-d+1}}-\cos ^{2} \frac{2 \pi}{d-s^{-d+1}}\right)=4 s^{2}\left(\sin ^{2} \frac{2 \pi}{d-s^{-d+1}}-\sin ^{2} \frac{2 \pi}{d+\frac{e}{2} s^{-d+1}}\right)= \\
& =4 s^{2}\left(\sin \frac{2 \pi}{d-s^{-d+1}}-\sin \frac{2 \pi}{d+\frac{e}{2} s^{-d+1}}\right)\left(\sin \frac{2 \pi}{d-s^{-d+1}}+\sin \frac{2 \pi}{d+\frac{e}{2} s^{-d+1}}\right)=
\end{aligned}
$$




$$
\begin{aligned}
&= 16 s^{2} \sin \left(\frac{\pi}{d-s^{-d+1}}-\frac{\pi}{d+\frac{e}{2} s^{-d+1}}\right) \cos \left(\frac{\pi}{d-s^{-d+1}}+\frac{\pi}{d+\frac{e}{2} s^{-d+1}}\right) . \\
& \cdot \sin \left(\frac{\pi}{d-s^{-d+1}}+\frac{\pi}{d+\frac{e}{2} s^{-d+1}}\right) \cos \left(\frac{\pi}{d-s^{-d+1}}-\frac{\pi}{d+\frac{e}{2} s^{-d+1}}\right)< \\
&<16 s^{2} \pi^{2}\left(\frac{1}{\left(d-s^{-d+1}\right)^{2}}-\frac{1}{\left(d+\frac{e}{2} s^{-d+1}\right)^{2}}\right)=\frac{16 \pi^{2}\left(\frac{e}{2}+1\right) s^{-d+3}\left(2 d+\left(\frac{e}{2}-1\right) s^{-d+1}\right)}{\left(d-s^{-d+1}\right)^{2}\left(d+\frac{e}{2} s^{-d+1}\right)^{2}} .
\end{aligned}
$$

Since $\left(d-s^{-d+1}\right)^{2}>(d-1)^{2}>2 d+1>2 d+\left(\frac{e}{2}-1\right) s^{-d+1}$, it is suffices to prove that $d+\frac{e}{2} s^{-d+1}>4 \pi \sqrt{\frac{e}{2}+1} s^{\frac{-d+3}{2}}$. Using $k \geq e+2 \geq 4$ and $d \geq 7$, we obtain

$$
s^{\frac{d-3}{2}}\left(d+\frac{e}{2} s^{-d+1}\right)>(k-1) d \geq(e+1) d>4 \pi \sqrt{\frac{e}{2}+1} .
$$

\section{References}

[1] E. Bannai and T. Ito. On finite Moore graphs. J. Fac. Sci. Tokyo Sect. 1A, 20 (1973) 191-208.

[2] E. Bannai and T. Ito. Regular graphs with excess one. Discrete Math. 37 (1981) 147-158.

[3] N. L. Biggs. Algebraic Graph Theory. Cambridge University Press, Second Edition, Great Britain (1993)

[4] N. L. Biggs and T. Ito. Graphs with even girth and small excess. Math. Proc. Camb. Philos. Soc. 88 (1980) 1-10.

[5] C. Dalfó, E. R. van Dam, M. A. Fiol, E. Garriga and B. L. Gorissen. On almost distance-regular graphs. J. Combin. Theory Ser. A 118 (2011) 1094-1113.

[6] E. V. Damm, W. H. Haemers. Which graphs are determined by their spectrum? Linear Algebra Appl. (2003) 241-272

[7] R. M. Damerell. On Moore graphs. Proc. Cambridge Phil. Soc. 74 (1973) 227-236.

[8] P. Erdős and H. Sachs. Reguläre Graphen gegebener Taillenweite mit minimaler Knotenzahl. Wiss. Z. Uni. Halle (Math. Nat.) 12 (1963) 251-257.

[9] G. Exoo and R. Jajcay. Dynamic cage survey. Electron. J. Combin., Dynamic Survey 16, September 2008.

[10] S. Filipovski. On bipartite cages of excess 4. Electron. J. Combin. 24(1) (2017) \#P1.40.

[11] C. D. Godsil, B. D. McKay. Feasibility conditions for the existence of walk-regular graphs. Linear algebra and its applications. 30 (1980) 5161. 
[12] T. B. Jajcayová, S. Filipovski and R. Jajcay. Improved lower bounds for the orders of even-girth cages. Electron. J. Combin. 23(3) (2016) \#P3.55.

[13] G. Pineda-Villavicencio. Non-existence of bipartite graphs of diameter at least 4 and defect 2. Journal of Algebraic Combinatorics 34 (2011), no. 2, 163-182.

[14] H. Sachs. Regular graphs with given girth and restricted circuits. J. London Math. Soc. 38 (1963) 423-429.

[15] R. C. Singleton. On minimal graphs of maximum even girth. J. Combin. Theory 1 (3) (1966) 306-332.

[16] W. T. Tutte. A family of cubical graphs. Proc. Cambridge Philos. Soc. 43 (1947). 